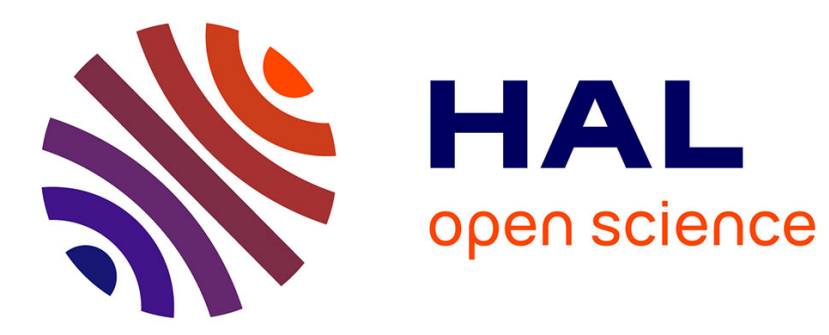

\title{
A blueprint of seed desiccation sensitivity in the genome of Castanospermum australe
}

\author{
Alexandre Marques, Maria-Cecilia D Costa, Jill M Farrant, Henk Hilhorst, \\ Julia Buitink, Wilco Ligterink, Olivier Leprince, Sandra Pelletier, Toni \\ Gabaldon, M. Eric Schranz, et al.
}

\section{To cite this version:}

Alexandre Marques, Maria-Cecilia D Costa, Jill M Farrant, Henk Hilhorst, Julia Buitink, et al.. A blueprint of seed desiccation sensitivity in the genome of Castanospermum australe. BioRxiv, 2019, 10.1101/665661 . hal-02624811

\section{HAL Id: hal-02624811 https://hal.inrae.fr/hal-02624811}

Submitted on 26 May 2020

HAL is a multi-disciplinary open access archive for the deposit and dissemination of scientific research documents, whether they are published or not. The documents may come from teaching and research institutions in France or abroad, or from public or private research centers.
L'archive ouverte pluridisciplinaire HAL, est destinée au dépôt et à la diffusion de documents scientifiques de niveau recherche, publiés ou non, émanant des établissements d'enseignement et de recherche français ou étrangers, des laboratoires publics ou privés. 
A blueprint of seed desiccation sensitivity in the genome of Castanospermum australe

Alexandre Marques ${ }^{1 \dagger}$, Maria-Cecília D. Costa ${ }^{2 \dagger}$, Udisha Chathuri ${ }^{2}$, Eef Jonkheer ${ }^{3,4}$, Tao Zhao $^{4}$, Elio Schijlen ${ }^{5}$, Martijn Derks ${ }^{3}$, Harm Nijveen ${ }^{3}$, Marina Marcet-Houben ${ }^{6,7}$, Irene Julca $^{6,7}$, Julien Delahaie ${ }^{8}$, M. Eric Schranz ${ }^{4}$, Toni Gabaldon ${ }^{6,7,9}$, Sandra Pelletier ${ }^{8}$, Olivier Leprince ${ }^{8}$, Wilco Ligterink ${ }^{1}$, Julia Buitink*8 ${ }^{* 8}$ Henk W.M. Hilhorst*1, Jill M.

Farrant*2

${ }^{1}$ Laboratory of Plant Physiology, Wageningen University and Research, Wageningen, The Netherlands; ${ }^{2}$ Department of Molecular and Cell Biology, University of Cape Town, Private Bag, Rondebosch 7701, South Africa; ${ }^{3}$ Bioinformatics Group, Wageningen University and Research, Wageningen, The Netherlands; ${ }^{4}$ Biosystematics Group, Wageningen University and Research, Wageningen, The Netherlands; ${ }^{5}$ Bioscience, Wageningen Plant Research International, Wageningen, The Netherlands; ${ }^{6}$ Centre for Genomic Regulation (CRG), The Barcelona Institute of Science and Technology, Dr. Aiguader 88, Barcelona 08003, Spain; ${ }^{7}$ Universitat Pompeu Fabra (UPF), Barcelona 08003, Spain; ${ }^{8}$ Institut de Recherche en Horticulture et Semences, UMR1345, INRA, Agrocampus Ouest, Université d'Angers, SFR 4207 QASAV, 49071 Beaucouzé, France; ${ }^{9}$ ICREA, Pg. Lluís Companys 23, Barcelona 08010, Spain.

${ }^{\dagger}$ These authors contributed equally to this work

*Corresponding authors

Author for correspondence:

Henk W.M. Hilhorst

Tel: +31317483646

Email: henk.hilhorst@wur.nl 


\section{Summary}

- Most angiosperms produce seeds that are desiccated on dispersal with the ability to retain viability in storage facilities for prolonged periods. However, some species produce desiccation sensitive seeds which rapidly lose viability in storage, precluding ex situ conservation. Current consensus is that desiccation sensitive seeds either lack or do not express mechanisms necessary for the acquisition of desiccation tolerance.

- We sequenced the genome of Castanospermum australe, a legume species producing desiccation sensitive seeds, and characterized its seed developmental physiology and transcriptomes.

- C. australe has a low rate of evolution, likely due to its perennial life-cycle and long generation times. The genome is syntenic with itself, with several orthologs of genes from desiccation tolerant legume seeds, from gamma whole-genome duplication events being retained. Changes in gene expression during development of $C$. australe seeds, as compared to desiccation tolerant Medicago truncatula seeds, suggest they remain metabolically active, prepared for immediate germination.

- Our data indicates that the phenotype of $C$. australe seeds arose through few changes in specific signalling pathways, precluding or bypassing activation of mechanisms necessary for acquisition of desiccation tolerance. Such changes have been perpetuated as the habitat in which dispersal occurs is favourable for prompt germination.

Key words: desiccation-sensitivity, evolution, orthodox, phylome, recalcitrant, seed development, synteny

\section{Introduction}

Seeds of most gymnosperm and angiosperm species are shed in the desiccated state and can be stored dry under sub-zero temperatures for prolonged periods of time, thus facilitating plant germplasm conservation. Such desiccation tolerant (DT) seeds are termed 'orthodox'. However, seeds of some species are desiccation-sensitive (DS), also referred to as 'recalcitrant', and cannot be successfully stored under typical conditions (Berjak \& Pammenter, 2013). DS-seeded species are mostly found in the humid tropics and may 
represent up to $50 \%$ of the species present in tropical evergreen rain forests (Hamilton et al., 2013). Such species occur in environments conducive to immediate seed germination and thus selective pressure for desiccation tolerance has been relaxed or is absent. It has been hypothesized that seed desiccation sensitivity is a derived trait that evolved independently in non-related clades (Berjak \& Pammenter, 2000). Genes responsible for seed desiccation tolerance would have been lost, repressed and/or mutated in DS seeded species (Berjak \& Pammenter, 2008). However, this hypothesis remains to be tested at the genome level. Here we applied an extensive phylogenetic comparison to obtain a genomic blueprint of desiccation sensitivity in seeds.

Desiccation tolerance is acquired mid-way during the development of orthodox seeds when seed filling is approximately half-way, corresponding to a steep drop in water content of the seeds, concomitantly with a transient rise in abscisic acid (ABA) content (Bewley et al., 2013). This acquisition comprises highly coordinated molecular events, including the repression of photosynthesis and energy metabolism, and accumulation of protective components, such as late embryogenesis abundant (LEA) proteins, anti-oxidants, and soluble sugars (Leprince et al., 2017). These events are tightly regulated by hormones such as ABA and transcription factors (TFs) such as ABSCISIC ACID INSENSITIVE 3 (ABI3), FUSCA 3 (FUS3) and LEAFY COTYLEDON 1 (LEC1) (Leprince et al., 2017). Conversely, in the development of DS seeds, the acquisition of desiccation tolerance and accumulation of the above-mentioned protectants appear to be suppressed and rather they directly progress towards germination (Farrant et al., 1993b; Francini et al., 2006; Delahaie et al., 2013). However, the genetic makeup underlying the DS seed phenotype is unknown.

The legume family (Fabaceae) contains many agriculturally important species, all producing DT seeds, such as soybean (Glycine max), the common bean (Phaseolus vulgaris), lentils (Lens culinaris) and chickpeas (Cicer arietinum). Moreover, legume species, such as soybean and Medicago truncatula, are important experimental models for molecular and physiological studies on seed desiccation tolerance (Chatelain et al., 2012; Delahaie et al., 2013; Verdier et al., 2013; Zinsmeister et al., 2016). In addition, the genomes of 16 DTseeded legume species have been sequenced. Thus, a large amount of information is available, allowing comparative analysis among them.

Castanopermum australe A. Cunn \& C. Fraser ex Hook, also known as the Moreton Bay Chestnut or Blackbean, is a leguminous tropical tree native to the east coast of Australia and west Pacific islands. In contrast to most legume species, C. australe produces DS seeds. It is the only known species in the genus that forms a separate and early branching clade within 
the Papilionoideae subfamily (Cardoso et al., 2012). The earliest-branching papilionoids fall within an ADA clade, which includes the monophyletic tribes Angylocalyceae, Dipterygeae, and Amburanae. C. australe's important phylogenetic position in the basis of the ADA clade (Angylocalyaceae) makes its genome ideal to study both trait evolution and the ancient polyploid history of papilionoid legumes (Schranz et al., 2012).

Here, we provide detailed genomic sequence information of $C$. australe combined with time-resolved gene expression analysis of seed development of this species, including the comparison with other species producing either DS or DT seeds. Such information is key to understanding mechanisms of desiccation tolerance and, ultimately, to design strategies to improve tolerance of extreme water loss in DS seeds for conservation purposes. We investigated genomic changes associated with seed desiccation sensitivity, including gene deletions, severe mutations and gene mis-expression, as well as their relationship with gene expression patterns during seed development and maturation.

\section{Materials and Methods}

Plant material

A population of trees of C. australe growing in Pietermaritzburg (Kwazulu-Natal Province, South Africa) was the source of plant material for this work. Seed development occurs over a 6-month period, during which pods were harvested weekly. Seeds were extracted and, following histodifferentiation, were separated into component tissues (axis, cotyledons and seed coat). The following was determined annually over 2 seasons. Whole seed mass and that of component tissues $(n=60)$ and water content $(n=10-20)$ was determined gravimetrically by oven drying. The ability of intact seeds to germinate was tested by planting in vermiculite.

Once germinable, the amount of water loss tolerated by axes and cotyledons was determined by flash drying (Berjak et al., 1990). Axis survival was determined as the ability to produce both shoots and roots when cultured in vitro on full strength MS medium. Survival of cotyledons was assessed by tetrazolium staining followed by spectrometric analysis (Sershen et al., 2012). Critical water contents (calculated on a $\mathrm{gH}_{2} \mathrm{O}_{\mathrm{g}} \mathrm{g}^{-1}$ dry mass) were calculated at those stages at which $50 \%$ survival was observed.

Sugar and ABA content and determination 
Sugars were extracted from frozen and lyophilised seeds and analysed by HPLC on a Carbopac PA-1 column (Rosnoblet et al., 2007) (Dionex Corp., Sunnyvale, CA, USA). Three independent extractions and assays were performed on approx. $100 \mathrm{mg}$ of tissue.

ABA was extracted and quantified as described by (Floková et al., 2014).

Genome sequencing and assembly

Freeze-dried leaf material was used for DNA isolation as described by (Bernatzky \& Tanksley, 1986) with modifications. The genomic C. australe library consisted of 30x coverage PacBio with a mean read length of $7.8 \mathrm{~Kb}$. In addition, an Illumina paired end library with reads of $100 \mathrm{bp}$ and a 200-400 bp insert size was constructed and sequenced to a $64 x$ coverage. Reads originating for contaminants were removed from all sequence data prior to assembly. Organelle genomes were also removed from the main genome assembly. Illumina reads were error-corrected using Lighter (Song et al., 2014) and assembled using SparseAssembler (Ye et al., 2012). A hybrid assembly was produced with DBG2OLC (Ye et $a l ., 2016)$ and the contigs were reordered and connected into scaffolds using SSPACELongRead (Boetzer \& Pirovano, 2014). The assembly was polished using Sparc (Ye et al., 2012) and Pilon (Walker et al., 2014). PBJelly2 (English et al., 2012) was used for gap closure and genome improvement. Alignments due to gene duplication and repeats were filtered out using the delta-filter utility of the MUMmer package (Kurtz et al., 2004). The assembly was validated by mapping the available RNA and DNA libraries to the genome with Bowtie2 (Langmead \& Salzberg, 2012) and Blasr (Chaisson \& Tesler, 2012). Assembly statistics were calculated using QUAST (Gurevich et al., 2013). Gene space completeness was measured using BUSCO (Benchmarking Universal Single-Copy Orthologs (Simão et al., 2015)). The MAKER2 annotation pipeline (Holt \& Yandell, 2011) was applied for gene prediction and repeat annotation.

\section{Transcriptome analysis}

C. australe seeds were harvested at weekly intervals after seed set and at shedding from trees growing in Pietermaritzburg in 2009 and 2011. Six developmental stages were collected for cotyledon tissues, and three stages for the embryonic axes. Prior to maturation, when embryos were small, cotyledon tissue was used. For the green pod stage (Figure 2), axes and cotyledons were separated. Transcriptome analysis was performed on newly designed 12x135K Nimblegen arrays (IRHS_Ca_102K_v1) for C. australe, based on the genome 
assembly of (Delahaie et al., 2013). RNA amplification, labelling and hybridization was performed according to (Terrasson et al., 2013). Four biological replicates were analysed per developmental stage using the dye-swap method, and statistical analysis on the gene expression data was performed according to (Verdier et al., 2013). Data are deposited in the NCBI Gene Expression Omnibus database (accession no. GSE109217, samples GSM2935461-GSM2935508). A gene was considered differentially expressed if $\mathrm{P} \leq 0.05$ in at least one comparison (axis or cotyledon) after the application of linear modelling.

Over-representation analysis (ORA) was used to recover over-represented biological processes using the app BiNGO (default settings) (Maere et al., 2005) for Cytoscape.

Orthologs were defined as hits with lowest Expect value (E-value) observing a threshold of $<10^{-10}$. Multiple hits were considered orthologs when the difference between their E-values and the lowest hit's E-value was smaller than $10^{-10}$.

Members of the eight LEA protein families were identified uploading Hidden Markov Models (HMM) for each family from the PFAM database (Finn et al., 2011) to HMMER 3.1b2 package (Eddy, 2011). All proteins with significant hits (E-value $\leq 0.01)$ were selected.

Phylome reconstruction

We reconstructed three phylomes, one for a species set closely related to C. australe (phylome 110) and starting in C. australe, a second one based on a broader taxonomic focus also starting in C. australe (111) and a third one also broad but starting in Fragaria vesca (112). The phylome 112 was used to search for lost genes in C. australe. It starts in F. vesca that is the closest outgroup DT seeded species with a high-quality genome sequence. Both phylomes were reconstructed using the same approach (Huerta-Cepas \& Gabaldon, 2011). All data generated during the phylome reconstruction has been deposited in phylomeDB (PMID:24275491) under the phylomeID codes 110 and 111. The trees, alignments and orthology and paralogy predictions are accessible to browse or download at the PhylomeDB database (Huerta-Cepas et al., 2014). A set of 183 one-to-one orthologous proteins present across the compared species was used to reconstruct a species phylogeny.

Whole genome duplication analysis

The $C$. australe genome was compared to genomic data from five Legumes: Glycine max, Lotus japonicus, Medicago truncatula, Phaseolus vulgaris, Trifolium pratense. G. max and 
M. truncatula are tetraploids and the rest are diploid species. The diploid Fragaria vesca species, of the family Rosaceae, was selected as outgroup as it is one of the closest relatives with a completed genome available outside of the Legume family. The assemblies of: G. max, L. japonicus, and M. truncatula are on chromosome level which made it easier to identify genome collinearity and duplication patterns.

Synteny analysis

A synteny network approach (Zhao et al., 2017) was implemented to compare the synteny of C. australe and other whole-genome sequenced legume species available at the Legume Information System (https://legumeinfo.org). BLASTP was used for pairwise genome comparison. MCScanX (Wang et al., 2012) was used for synteny block detection. Infomap algorithm (Rosvall \& Bergstrom, 2008) implemented in R igraph package was used for synteny network clustering. Clusters containing genes/nodes from more than 8 (out of the 10) legume species but no $C$. australe node(s) were screened out for further investigation. The maximum distance between two matches was 20 genes, a syntenic block consists of minimum 5 genes and no blocks were merged. Quota Align was enabled to determine the syntenic depth (the number of times a genomic region is syntenic). For calculating the fractionation bias, the window size was lowered from 100 to 25 considering the smaller contigs of the $C$. australe genome. Synonymous (Ks) and non-synonymous (Kn) site mutations were calculated for each syntenic gene pair. Mutation rates were used to determine if genes were duplicated by the WGD event or not. The distribution of the Ks rate was used to set a different cut-off per species.

\section{$\mathrm{dN} / \mathrm{dS}$ analysis}

We used the genome of 5 legume species from phytozome (M. truncatula, G. max, Glycine soja, T. pratense and P. vulgaris) and C. australe. The SynMap tool in the online CoGe portal was used to find syntenic gene pairs within these species. We set the DAGChainer on a maximum distance between two matches for 50 genes and minimum number of aligned pairs on 3 genes. To establish sets of orthologous among the 5 species against M. truncatula, the method of reciprocal best hits using Last was used.

Codeml in the Phylogenetic Analysis by Maximum Likelihood (PAML) package was used to estimate the $\mathrm{dN}$ (the rate of non-synonymous substitutions), $\mathrm{dS}$ (the rate of 
synonymous substitutions) and the ratio of $d N / d S$ (Yang, 2007). Orthologs with $d S>5, d N>2$ or $\mathrm{dN} / \mathrm{dS}>2$ were filtered. For genes with multiple syntelogs we kept the pair with the lowest $\mathrm{dN} / \mathrm{dS}$.

\section{Results}

Castanospermum australe seed development

Seed development in C. australe occurs over a period of 6 months, with reserve accumulation and maximum embryo size completed approximately 3 months after flowering and coincident with pods becoming yellow (Figure 1 and Table 1). Unlike the embryo, the seed coat declined in mass until just prior to the yellow-green pod stage, with no further decline once embryos reached full size. Water content declined in all tissues once reserve accumulation was complete at the yellow pod stage. This loss stabilized in all tissues but the seed coat, which continued to lose water.

While seeds had the capacity to germinate prior to reaching full embryonic size, germination rate was slow. Full germination capacity, typically of newly shed seeds was achieved at the yellow green pod stage (Table 1). The lethal water content of axes below which $50 \%$ viability was lost after drying declined from $0.45 \mathrm{gH}_{2} \mathrm{O} / \mathrm{g}^{-1}$ dry mass in those extracted from green pods, to $0.23 \mathrm{gH}_{2} \mathrm{O} / \mathrm{g}^{-1}$ dry mass in axes from yellow pods, after which there was no significant change. Cotyledons were more sensitive to dehydration, with $50 \%$ loss of viability occurring below $0.82 \mathrm{gH}_{2} \mathrm{O} / \mathrm{g}^{-1}$ dry mass in those from green pods, this declining slightly with development to $0.70 \mathrm{gH}_{2} \mathrm{O} / \mathrm{g}^{-1}$ dry mass in cotyledons from brown pods (Table 1).

ABA regulates many aspects of plant growth and development including embryo maturation, seed dormancy, germination, cell division and elongation. ABA content of $C$. australe embryos was high during early developmental stages but declined considerably in both axes and cotyledons in the transition from the yellow-green to the yellow pod stage (Table 1). ABA content increased considerably in the seed coat in the transition from the yellow to the brown pod stage, especially in the point of attachment (tissue that attaches the embryo to the pod). 
Seed maturation drying

DT seeds become tolerant of drying midway during seed development, concomitant with reserve accumulation (Chatelain et al., 2012). From this stage onwards, there is progressive loss of water characterizing a process termed 'maturation' drying, that occurs after reserve accumulation is complete and full seed size is attained. There was no maturation drying typical of DT seeds after the yellow pod stage in C. australe (Table 1).

Previous work has identified transcripts that accumulate during the acquisition of desiccation tolerance in M. truncatula seeds (Terrasson et al., 2013; Righetti et al., 2015). Homologs of 121 of these genes failed to accumulate transcripts to a similar extent in $C$. australe cotyledons in comparable seed developmental stages (Table S1). These transcripts are related to sugar metabolism, photosynthesis, seed development, protection against abiotic stress and modulation of plant stress responses. Examples include $A B I 3, A B I 5$, chaperone proteins, heat shock factor proteins, putative LEAs, transparent testa protein, oleosins, 1 CYSTEINE PEROXIREDOXIN, and $\alpha$-galactosidases.

We identified 269 transcripts with decreasing abundance in C. australe during final maturation and increasing abundance in M. truncatula (Table S2). A certain number of these transcripts are possibly involved in longevity (life span in the dried state) (Verdier et al., 2013; Righetti et al., 2015). Some of these genes are related to metabolic and catabolic processes, such as lipid metabolic process, cellular lipid metabolic process and nitrogen compound metabolic process (Table S3), reflecting the type of reserves accumulated. Mature C. australe seeds predominantly accumulate starch (85\% dry mass, Table1). Lipids and proteins constitute only 3-6\% and 2.8\% dry mass, respectively. Mature seeds of $M$. truncatula predominantly accumulate protein (30-40\% dry mass), but also contain storage lipids (7-9\% dry mass) and a small amount of starch (<1\% dry mass) (Djemel et al., 2005).

We also identified 296 transcripts with decreasing abundance in M. truncatula and increasing abundance in C. australe. They are related to root development, developmental process and regulation of localization (Table S2), which are likely associated with germination related processes.

LEA proteins have been related to survival in the dry state (Chatelain et al., 2012) and to responses to environmental stresses, including desiccation (Tunnacliffe \& Wise, 2007). In C. australe, several LEA proteins failed to accumulate in cotyledons (Delahaie et al., 2013). A genome-wide search for LEAs in the genome of $C$. australe identified 94 LEA motifcontaining proteins, a number similar to what has been described for DT-seeded species, for 
example, 88 in Arabidopsis thaliana and 99 in Sorghum bicolor, as well as for vegetative DT in the resurrection plants Oropetium thomaeum and Xerophyta viscosa with 94 and 126 LEAs, respectively (VanBuren et al., 2017; Costa et al., 2017). A hierarchical clustering of expression of the LEAs in developing seeds of $C$. australe and $M$. truncatula separated the transcripts in two major clusters (Figure S1). LEAs in the first cluster belong to different families and transcript abundance increased considerably in M. truncatula seeds towards mid maturation. However, in C. australe most of these increased only slightly in early stages of development but declined during the later stages. LEAs in the second cluster belong to the LEA_2 family and do not undergo major changes in transcript abundance in either species. This family encodes 'atypical' LEA proteins because of their more hydrophobic character compared to other LEA families (Hundertmark \& Hincha, 2008). Functional studies on LEA_2 proteins suggest that they do not act in the protection of membranes in tissues undergoing dehydration, although some proteins of this family were shown to have enzyme protective properties under both freezing and drying conditions (Dang et al., 2014).

Changes in soluble sugar content and composition have been described as a characteristic of late maturation in DT seeds and correlate with the acquisition of longevity and preparation for the dry state (Wang et al., 2013; Leprince et al., 2017). Whereas in all DT legume seeds, raffinose family oligosaccharides (RFO) are the predominant sugars that increase during late maturation, $C$. australe seeds were composed of 7-10\% of soluble sugars, with sucrose being the most abundant sugar detected and only minute amounts $(0.7 \%$ of total soluble sugars at the brown pod stage) of RFOs accumulating. Low ratios of sucrose:RFO accumulation have been proposed to be a signature of desiccation tolerance and potential indicators of seed storage categories (Steadman et al., 1996; Farrant et al., 2012) and the opposite of this, as depicted in C. australe could be one of the reasons for desiccation sensitivity in this species.

\section{Genome sequencing and assembly}

In an effort to obtain a genomic blueprint of DS seeds we sequenced the genome of $C$. australe. This species has a key position in the legume family phylogenetic tree, in the basis of the ADA clade, which favours the study of trait evolution as well as the ancient polyploid history of papilionoid legumes.

We produced an assembly with a total length of $382 \mathrm{Mb}$ and an N50 of $832.6 \mathrm{~Kb}$ that covers $96.7 \%$ of the predicted genome size. The assembly consisted of 1,210 contigs and 
1,027 scaffolds (Table 2). The GC content was 32.9\%. Genome annotation identified 29,124 protein-coding genes of which $98.1 \%$ show high sequence similarity to proteins in TrEMBL and $84.4 \%$ in Swiss-Prot databases. An estimation of genome completeness indicated that 96.4\% of the BUSCO (Benchmarking Universal Single-Copy Orthologs) genes were present. Transposable elements covered $15.5 \%$ of the total genome. Repeat elements comprised 119 $\mathrm{Kb}$ of SINE (Short Interspersed Nuclear Element) retrotransposons, $383 \mathrm{~Kb}$ of LINE (Long Interspersed Elements) retrotransposons, $13 \mathrm{Mb} \mathrm{DNA}$ transposons and $42 \mathrm{Mb}$ of annotated LTR (long terminal repeat-retrotransposons) sequences (Table 2).

\section{Genomic alterations linked to seed desiccation sensitivity}

To investigate the evolution of $C$. australe and legume diversification, a phylome was constructed. A phylome constitutes the collection of all gene phylogenies in a genome. It is a valuable source of information to establish evolutionary relationships among organisms and their genes (Huerta-Cepas et al., 2014). Our phylome contained the evolutionary histories of all C. australe protein coding genes and their homologues in 20 publicly available sequenced plant species (Figure 2). These species represent a broad phylogenetic distribution and include the diverse seed storage phenotypes, i.e. DT, DS and intermediate seeds. Intermediate seeds are typically tolerant of relatively extreme water loss, to $0.1-0.14 \mathrm{~g} \mathrm{H}_{2} \mathrm{O} / \mathrm{g}^{-1}$ dry mass but no lower than this(Marques et al., 2018), and have poor survival under conventional storage conditions (Berjak \& Pammenter, 2013). Such seeds thus show a storage phenotype inbetween orthodox and recalcitrant seeds.

The phylome analysis indicated that very few protein-coding genes $(\leq 1 \%)$ were present in DS species only, and no protein-coding genes were retained in all DT species but lost in all DS species (Figure 2). Several genes were lost in all DS species and retained in at least half of the DT species (Table S4). 76 genes lost their ortholog but kept a paralog in $C$. australe and 59 genes were lost in C. australe without detected paralogs, of which 11 were shared with other DS-seeded species.

According to the phylome, 3,716 gene families were expanded exclusively in $C$. australe, of which 180 were associated with transposons. Expansion size ranged from 2 to 32 genes and involved $30.5 \%$ of the predicted proteome. After removal of expansions associated with transposable elements or viruses, the remaining expanded gene families were enriched for GO (gene ontology) terms such as defence response, flavonoid biosynthetic process, terpene synthase activity and nutrient reservoir activity. GO terms associated with terpene 
synthase activity, lyase activity and pectinesterase activity were also enriched at the base of the Papilionoideae subfamily. The phylome analysis also indicated that the duplication frequency at the base of the Papilionoideae subfamily is lower (0.22) than that found at the base of the Fabaceae family (0.73, Figure 3$)$. Histograms of the synonymous rates and average rates of syntenic blocks for six legume species showed distinctive peaks tracing back to the shared papilionoid legume whole genome duplication (WGD), suggesting that the rate of evolution is very diverse among closely related family members (Figure 4). The peak in $C$. australe corresponds to a rate of synonymous (Ks) site mutations of 0.25 which is less than half of the rate observed in G. $\max (0.6)$ and a third of that in M. truncatula (0.85). The substitution rate in C. australe is so low that a second peak corresponding to the eudicot hexaploidy (gamma WGD event) is still visible. The gamma event was also detected in the histograms of block averages in G. max and P. vulgaris. The low Ks rate in C. australe is likely due to this species being the only perennial in this comparative study and the one with the longest generation times.

The evolution of a trait is shaped by the selective pressures to which it is subject. Some selective pressures act to increase the benefits accumulated while others act to reduce the costs incurred, affecting the cost/benefit ratio. Different selective pressures can be estimated by the ratio of the number of nonsynonymous substitutions per non-synonymous site $(\mathrm{dN})$ in a specific period to the number of synonymous substitutions per synonymous site (dS) in the same period (Mugal et al., 2014).

Genome wide analysis of protein coding genes of $C$. australe in comparison with other legume genomes enabled the identification of genes with 2 -fold higher $\mathrm{dN} / \mathrm{dS}$ in $C$. australe (Table S5). Among these were genes associated with hormonal signalling, such as ACTIVATION-TAGGED BRI1 (BRASSINOSTEROID-INSENSITIVE1)-SUPPRESSOR1 (ATBS1), Ethylene Insensitive 3 family protein, BRI1-ASSOCIATED RECEPTOR KINASE (BAK1) and SALT TOLERANCE HOMOLOG2 (STH2). Other examples are: SEEDSTICK (STK), TRANSPARENT TESTA5 (TT5), ENDO-BETA-MANNANASE7 (MAN7), FLOWER FLAVONOID TRANSPORTER (FFT) and ROTUNDIFOLIA3 (ROT3).

The evolution of a trait can also be investigated by analysing the degree to which genes remain on corresponding chromosome (synteny) and in corresponding orders over time. We investigated whether the loss of synteny in $C$. australe genes could be related to the loss of seed desiccation tolerance. There are 169 genes re-arranged in the $C$. australe genome that have syntenic orthologs in 50 angiosperm species. Most noteworthy among these were the genes BRASSINOSTEROID INSENSITIVE 3 and 5 (BIN3 and BIN5), which participate in 
brassinosteroid (BR) signalling and are associated with seed size (Yin et al., 2002). Furthermore, genes such as MINISEED3 (MINI3) and HAIKU1 (IKU1), regulators of seed size via the BR pathway in A. thaliana (Luo et al., 2005), also lost synteny in C. australe, which could contribute to the large seed size in this species.

The synteny between the genome of $C$. australe and other legume species was evaluated by aligning the genome of $C$. australe against itself and against the genomes of $G$. max and M. truncatula (Figure S2). Most of the C. australe genome is syntenic with itself and mostly duplicated after the WGD event. While the duplicates are associated with the most recent WGD event, many paralogs derived from the gamma event were also detected. The alignment of $C$. australe against $G$. max indicated a high amount of syntenic orthologs and paralogs, whereas the alignment of $C$. australe against $M$. truncatula indicated that although many syntenic orthologs have been conserved, most of the WGD-derived paralogs were lost. Moreover, most of the duplicated regions retained by M. truncatula were also duplicated and retained in C. australe.

\section{Discussion}

In orthodox seeds, survival in the dry state is a result of a series of molecular and cellular processes that occur during the late stages of seed development. These processes result in the acquisition of desiccation tolerance and longevity in the dry state. Although we have a detailed understanding of these associated processes in orthodox seeds, limited information is available regarding the development of seeds that do not fully activate them, such as $C$. australe. Our study provides detailed information about $C$. australe seed development and desiccation sensitivity.

In $C$. australe axes and cotyledons, some water loss occurss during reserve accumulation but this stops once the embryo reaches its full size, stabilizing at 2.4 and 1.6 $\mathrm{gH}_{2} \mathrm{O} \mathrm{g}^{-1}$ dry mass, respectively (Table 1). This value is substantially higher than the 0.1 $\mathrm{gH}_{2} \mathrm{O} \mathrm{g}^{-1}$ dry mass reached by desiccation-tolerant seeds, such as $M$. truncatula.

The pattern of sugar accumulation also differs markedly between these species. The percentage of soluble sugars in seeds of $C$. australe (7-10\%) is comparable with the average percentage for legume species (8-10\% (Djemel et al., 2005)). However, while RFOs are the main sugars in M. truncatula, comprising $90 \%$ of the total soluble sugar content, in $C$. australe only minute amounts of RFOs, mainly stachyose, could be detected $(0.7 \%$ of total soluble sugars at the brown pod stage). Stachyose and raffinose contents were highest in seeds 
from the green pod stage and decreased with further progress of maturation (Table 1). A similar finding has been reported for the non-viviparous highly DS seeds of Avicennia marina (Farrant et al., 1992). In parallel, sucrose and glucose content increased. The reduction of stachyose content during further maturation suggests hydrolysis, normally occurring in germinating DT seeds (Rosnoblet et al., 2007). A comparative analysis of transcripts linked to RFO metabolism between C. australe and M. truncatula identified transcripts of genes related to the synthesis of sucrose from fructose- 6 phosphate that remained high in C. australe whereas they decreased in M. truncatula. Conversely, the transcripts of several genes related to the synthesis of galactinol or raffinose and stachyose accumulated during development of M. truncatula seeds while their abundance remained low in C. australe (Supplementary Figure S1B). This set of genes might explain the lack of RFO accumulation in C. australe seeds. Whereas the specific roles of RFOs in protection compared to the nonreducing sucrose remain unconfirmed, the DS A. thaliana abi3 mutants as well as Mt-abi5 are also impaired in the accumulation of RFOs (Zinsmeister et al., 2016).

At the transcriptome level, transcripts with decreasing abundance in M. truncatula and increasing abundance in $C$. australe reinforce the notion that towards the end of seed development, $C$. australe is metabolically active while $M$. truncatula is entering a phase of low metabolic activity and quiescence. Examples of these transcripts are beta-galactosidase (Medtr8g039160), xyloglucan galactosyltransferase (Medtr1g069460) and TCP family TF (Medtr6g015350). Interestingly, these genes lost synteny in $C$. australe compared to their $M$. truncatula orthologs. Their involvement in carbohydrate metabolism and control of cell proliferation hints at implication in the germination program. The germination program remains active in C. australe, as DS seeds generally do not display developmental arrest, allowing the maintenance of high metabolic activity. In contrast, transcripts of indole-3-acetic acid-amido synthetases, involved in auxin homeostasis, accumulated in M. truncatula during development but decreased in C. australe. Auxin has been reported to maintain seed dormancy by interacting with ABA (Liu et al., 2013).

At the genome level, very few protein-coding genes $(\leq 1 \%)$ were present in DS species only (Figure 2), supporting the hypothesis that independent evolutionary events gave rise to DS-seeded species. No protein-coding genes were retained in all DT species and lost in all DS species. However, several were lost in all DS and retained in at least half of the DT species. Among these were the transcription factors (TFs) VERDANDI and MYB44-like. VERDANDI participates in ovule identity complex and, when mutated, affects embryo sac 
differentiation in A. thaliana (Matias-Hernandez et al., 2010; Mendes et al., 2016). Interestingly, VERDANDI and MYB44-like were also retained in intermediate-seeded species. One gene (PLAC8) was lost without retention of paralogs in three out of four DSseeded species, namely C. australe, Castanea mollissima and Elaeis guineensis. The knockout of this gene caused increased seed and fruit size in maize (Libault \& Stacey, 2010). In addition, the fw 2.2 locus containing the PLAC8 gene has been suggested to be the key to the evolution of tomato fruit size (Frary, 2000). Large seeds and fruits are common features of DS species and presumably reduce the rate of seed drying and hence the risk of desiccationinduced embryo mortality (Daws et al., 2006).

Amongst the genes that lost synteny in C. australe without detected paralogs, 11 were shared with other DS-seeded species. Two of these genes, LEA2 and FIBRILLIN5 accumulate transcripts in M. truncatula during seed maturation and upon re-induction of desiccation tolerance in germinated seeds (Terrasson et al., 2013). The gene GUN5, a magnesium chelatase involved in retrograde signalling and ABA signalling to the nucleus (Jiang et al., 2014), was lost in C. australe without paralogs. This pathway is affected in M. truncatula abi5 mutants that produce seeds with strongly reduced longevity (Zinsmeister et al., 2016) and cannot reacquire desiccation tolerance after germination (Terrasson et al., 2013).

Examples of genes which lost their ortholog but kept a paralog in C. australe are RETARDED ROOT GROWTH-LIKE (RRL) and MOTHER OF FT (MFT), involved in ABAand BR-signalling. RRL mediates ABA signal transduction through ABI4 (Park et al., 2015) and MFT regulates seed germination and fertility involving ABA- and BR-signalling pathways (Sun et al., 2010).

ABA is involved in the formation of mature DT seeds, and inhibition of their subsequent germination under conditions unfavourable for seedling growth (Finkelstein, 2013). During seed development, an increase in ABA content has been related to a transition from growth by cell division to growth by cell enlargement and to cell cycle arrest at the G1/S transition. This increase may be related to the role of ABA in promoting senescence, a process which precedes abscission (Finkelstein, 2013). Additionally, the higher ABA content in the seed coat could play a role in delaying germination of the embryo until ideal conditions for germination are met, or to aid temporal and/or spatial dispersal without immediate loss of viability. Interestingly, the seed coat starts to peel away from the embryo in mature seeds (Figure 1) and this increases markedly once seeds are shed, suggesting increasing lack of inhibition of germination of the embryo. 
$\mathrm{ABA}$ is also a key regulator of abiotic stress responses and acquisition of desiccation tolerance during seed development (reviewed by (Dekkers et al., 2015)). Disruption of ABA biosynthesis or -signalling leading to lack of or insensitivity to ABA results in loss of seed desiccation tolerance (Verdier et al., 2013). We observed alterations in genes related to ABA signalling, such as MFT, GPCR-TYPE G PROTEIN 1 (GTG1), STH2, ABI3 and ABI5. C. australe lost an ortholog of MFT but maintained a paralog. Mutations in this gene may cause ABA hypersensitivity at germination and is associated with dormancy (Vaistij et al., 2013). STH2 is involved in ABA signalling, is highly expressed during embryogenesis (Xu et al., 2014) and has a high $\mathrm{dN} / \mathrm{dS}$ in C. australe. GTG1 was lost in C. mollissima and its knock-out causes ABA hyposensitivity in A. thaliana seeds (Pandey et al., 2009). ABI3 and ABI5 showed contrasting expression patterns during C. australe seed development compared to $M$. truncatula. These two TFs have been shown to play essential roles in seed development and acquisition of desiccation tolerance (Terrasson et al., 2013; Dekkers et al., 2015; Zinsmeister et al., 2016).

BRs have been implicated in seed development and are known to antagonize seed dormancy and stimulate germination (Steber, 2001). In C. australe, several genes involved in BR-biosynthesis and -signalling and seed development have undergone genetic changes (Figure 5). For example, BRASSINOSTEROID INSENSITIVE-LIKE 3 (BRL3) lost an ortholog but kept a paralog; IKU1 and MINI3 lost synteny; and ROT3, DE-ETIOLATED 2 (DET2), ATBS1 and BAK1 have higher dN/dS in C. australe than in M. truncatula. Furthermore, an ortholog of $A T B S 1$ was highly expressed during late development of $C$. australe contrasting with the decreasing expression in M. truncatula seeds. These data support the hypothesis that subcellular metabolism associated with germination is initiated during the late stages of development in the DS seeds of $C$. australe. Overall, our results support the hypothesis that the evolution of desiccation sensitivity was not caused by massive alterations in enzymes and structural proteins but instead by discrete mutations in regulatory genes.

Natural populations often undergo the weakening or removal of a selective force that had been important in the maintenance of a trait, characterizing a "relaxed selection" (Lahti et al., 2009). When a DT-seeded species is subjected to an environment where desiccation tolerance is not an adaptive trait, there should be relaxation of its evolutionary constraints that can eventually lead to its loss. DS-seeded species evolved in environments where the conditions favour immediate germination and seeds are programmed to initiate germination upon, or shortly after shedding (Farrant et al., 1993a; Daws et al., 2006; Berjak \& Pammenter, 2013). DT seeds, which very often display a form of dormancy, normally form seed banks in 
the soil. In contrast, DS seeds germinate immediately and usually form seedling banks under shaded forest canopy and take advantage of an eventual light gap for faster establishment. Furthermore, in these species, the generally increased seed size favours seedling establishment under shaded forest conditions (Daws et al., 2006).

In summary, seed desiccation sensitivity evolved multiple independent times in environments where water is highly abundant and predictable across long periods, favouring immediate seed germination. In such environments, the evolutionary pressure for DT seeds is relaxed and the production of DS seeds is not disadvantageous. This was the case for $C$. australe. Among the currently known DS-non-viviparous seeds, C. australe is one of the most sensitive to water loss. We have pinpointed some of the factors behind this sensitivity, namely displacements, loss of synteny and mis-expression of specific genes related to the BR- and ABA-signalling pathways, carbon metabolism, control of cell proliferation, protection against abiotic stresses and modulation of plant stress responses. These alterations are likely to have led to an increased seed size; high starch and low protein and lipid seed content; low accumulation of LEA proteins and RFOs in the seeds; and failure to start the seed maturation drying phase.

The low similarity between DS-seeded species confirms the hypothesis that desiccation sensitivity evolved independently. Moreover, it supports the idea that although the evolution of many factors was necessary for the appearance of seed desiccation tolerance, only a few changes in some of these factors are enough for its loss.

\section{Author contributions}

A.M. and M.-C.D.C. wrote the article; U.C. performed physiological experiments; M.-C.D.C., E.J., T.Z., M.D. and H.N. performed the bioinformatics; A.M., E.S., M.M.-H., I.J. and T.G. contributed to the genome and transcriptome analysis; J.D. and J.B. performed and analysed the transcriptomics; M.E.S. performed the PacBio sequencing and initial genome analysis; J.B., H.W.M.H. and J.M.F. initiated and coordinated the work and directed preparation of the article.

\section{Acknowledgements}

A.M. received financial support from CNPq-National Council for Scientific and Technological Development (246220/2012-0) Brazil. J.M.F. contributed towards this work from funding from the National Research Foundation (grant number 69416) and her DST-NRF South African Research chair (grant number 98406). This work was funded in part by a grant from the Region 
des Pays de la Loire, France (QUALISEM 2009-2013) and the bilateral Partenariat Hubert Curien (PHC) program France-South Africa (grant no. 25903RE) to O.L. and J.B.). We acknowledge David Lalanne and the ANAN platform of the SFR Quasav, Angers, France for the assistance with the microarray analysis. We acknowledge Bas te Lintel Hekkert for library preparation for genome sequencing.

\section{References}

Berjak P, Farrant JM, Mycock DJ, Pammenter NW. 1990. Recalcitrnat (homoiohydrous) seeds: the enigma of their desiccation-sensitivity. Seed Science and Technology 18: 297310.

Berjak P, Pammenter NW. 2000. What ultrastructure has told us about recalcitrant seeds. Revista Brasileira de Fisiologia Vegetal 12: 22-55.

Berjak P, Pammenter NW. 2008. From Avicennia to Zizania: Seed recalcitrance in perspective. Annals of Botany 101: 213-228.

Berjak P, Pammenter NW. 2013. Implications of the lack of desiccation tolerance in recalcitrant seeds. Frontiers in Plant Science 4: 1-9.

Bernatzky R, Tanksley SD. 1986. Toward a saturated linkage map in tomato based on isozymes and random cDNA sequences. Genetics 112: 887-98.

Bewley JD, Bradford KJ, Hilhorst HWM, Nonogaki H. 2013. Seeds. Physiology of development, germination and dormancy (JD Bewley, KJ Bradford, HWM Hilhorst, and H Nonogaki, Eds.). Springer.

Boetzer M, Pirovano W. 2014. SSPACE-LongRead: scaffolding bacterial draft genomes using long read sequence information. BMC Bioinformatics 15: 211.

Cardoso D, de Queiroz LP, Pennington RT, de Lima HC, Fonty É, Wojciechowski MF, Lavin M. 2012. Revisiting the phylogeny of papilionoid legumes: New insights from comprehensively sampled early-branching lineages. American Journal of Botany $\mathbf{9 9}$ : 1991-2013.

Chaisson MJ, Tesler G. 2012. Mapping single molecule sequencing reads using basic local alignment with successive refinement (BLASR): application and theory. $B M C$ Bioinformatics 13: 238.

\section{Chatelain E, Hundertmark M, Leprince O, Gall S Le, Satour P, Deligny-Penninck S,} Rogniaux H, Buitink J. 2012. Temporal profiling of the heat-stable proteome during late maturation of Medicago truncatula seeds identifies a restricted subset of late 
embryogenesis abundant proteins associated with longevity. Plant, Cell and Environment 35: $1440-1455$.

Costa M-CD, Artur MAS, Maia J, Jonkheer E, Derks MFL, Nijveen H, Williams B, Mundree SG, Jiménez-Gómez JM, Hesselink T, et al. 2017. A footprint of desiccation tolerance in the genome of Xerophyta viscosa. Nature Plants 3: 17038.

Dang NX, Popova A V., Hundertmark M, Hincha DK. 2014. Functional characterization of selected LEA proteins from Arabidopsis thaliana in yeast and in vitro. Planta 240: 325336.

Daws MI, Garwood NC, Pritchard HW. 2006. Prediction of desiccation sensitivity in seeds of woody species: A probabilistic model based on two seed traits and 104 species. Annals of Botany 97: 667-674.

Dekkers BJW, Costa MCD, Maia J, Bentsink L, Ligterink W, Hilhorst HWM. 2015. Acquisition and loss of desiccation tolerance in seeds: from experimental model to biological relevance. Planta 241: 563-577.

Delahaie J, Hundertmark M, Bove J, Leprince O, Rogniaux H, Buitink J. 2013. LEA polypeptide profiling of recalcitrant and orthodox legume seeds reveals ABI3-regulated LEA protein abundance linked to desiccation tolerance. Journal of Experimental Botany 64: 4559-4573.

Djemel N, Guedon D, Lechevalier A, Salon C, Miquel M, Prosperi JM, Rochat C, Boutin JP. 2005. Development and composition of the seeds of nine genotypes of the Medicago truncatula species complex. Plant Physiology and Biochemistry 43: 557-566.

Eddy SR. 2011. Accelerated profile HMM searches. PLoS Computational Biology 7.

English AC, Richards S, Han Y, Wang M, Vee V, Qu J, Qin X, Muzny DM, Reid JG, Worley KC, et al. 2012. Mind the gap: Upgrading genomes with Pacific Biosciences RS Long-Read sequencing technology. PLoS ONE 7: 1-12.

Farrant JM, Berjak P, Cutting JGM, Pammenter NW. 1993a. The role of plant growth regulators in the development and germination of the desiccation sensitive recalcitrant seeds of Avicennia marina. Seed Science Research 3: 55-63.

Farrant JM, Berjak P, Pammenter NW. 1992. Proteins in development and germination of a desiccation sensitive (recalcitrant) seed species. Plant Growth Regulation 11: 257-265.

Farrant JM, Cooper K, Nell H. 2012. Desiccation tolerance. Plant Stress Physiology: 238265.

Farrant JM, Pammenter NW, Berjak P. 1993b. Seed development in relation to desiccation tolerance: A comparison between desiccation-sensitive (recalcitrant) seeds of 
Avicennia marina and desiccation-tolerant types. Seed Science Research 3.

Finkelstein R. 2013. Abscisic acid synthesis and response. The Arabidopsis Book 11: e0166.

Finn RD, Clements J, Eddy SR. 2011. HMMER web server: Interactive sequence similarity searching. Nucleic Acids Research 39: 1-9.

Floková K, Tarkowská D, Miersch O, Strnad M, Wasternack C, Novák O. 2014. UHPLC-MS/MS based target profiling of stress-induced phytohormones. Phytochemistry 105: $147-157$.

Francini A, Galleschi L, Saviozzi F, Pinzino C, Izzo R, Sgherri C, Navari-Izzo F. 2006. Enzymatic and non-enzymatic protective mechanisms in recalcitrant seeds of Araucaria bidwillii subjected to desiccation. Plant Physiology and Biochemistry 44: 556-563.

Frary A. 2000. fw2.2: A quantitative trait locus key to the evolution of tomato fruit size. Science 289: 85-88.

Gurevich A, Saveliev V, Vyahhi N, Tesler G. 2013. QUAST: quality assessment tool for genome assemblies. Bioinformatics 29: 1072-1075.

Hamilton KN, Offord CA, Cuneo P, Deseo MA. 2013. A comparative study of seed morphology in relation to desiccation tolerance and other physiological responses in 71 Eastern Australian rainforest species. Plant Species Biology 28: 51-62.

Holt C, Yandell M. 2011. MAKER2: an annotation pipeline and genome-database management tool for second-generation genome projects. BMC Bioinformatics 12: 491.

\section{Huerta-Cepas J, Capella-Gutiérrez S, Pryszcz LP, Marcet-Houben M, Gabaldón T.} 2014. PhylomeDB v4: zooming into the plurality of evolutionary histories of a genome. Nucleic Acids Research 42: D897-D902.

Huerta-Cepas J, Gabaldon T. 2011. Assigning duplication events to relative temporal scales in genome-wide studies. Bioinformatics 27: 38-45.

Hundertmark M, Hincha DK. 2008. LEA (Late Embryogenesis Abundant) proteins and their encoding genes in Arabidopsis thaliana. BMC Genomics 9: 118.

Jiang S-C, Mei C, Wang X-F, Zhang D-P. 2014. A hub for ABA signaling to the nucleus: Significance of a cytosolic and nuclear dual-localized PPR protein SOAR1 acting downstream of Mg-chelatase H subunit. Plant Signaling \& Behavior 9: e972899.

Kurtz S, Phillippy A, Delcher AL, Smoot M, Shumway M, Antonescu C, Salzberg SL. 2004. Versatile and open software for comparing large genomes. Genome Biology 5: R12.

Lahti DC, Johnson NA, Ajie BC, Otto SP, Hendry AP, Blumstein DT, Coss RG, Donohue K, Foster SA. 2009. Relaxed selection in the wild. Trends in Ecology \& Evolution 24: 487-496. 
Langmead B, Salzberg SL. 2012. Fast gapped-read alignment with Bowtie 2. Nature methods 9: 357-9.

Leprince O, Pellizzaro A, Berriri S, Buitink J. 2017. Late seed maturation: Drying without dying. Journal of Experimental Botany 68: 827-841.

Libault M, Stacey G. 2010. Evolution of FW2.2-like (FWL) and PLAC8 genes in eukaryotes. Plant Signaling \& Behavior 5: 1226-1228.

\section{Liu S, Wang X, Wang H, Xin H, Yang X, Yan J, Li J, Tran LSP, Shinozaki K,} Yamaguchi-Shinozaki K, et al. 2013. Genome-wide analysis of ZmDREB genes and their association with natural variation in drought tolerance at seedling stage of Zea mays L. PLoS Genetics 9.

Luo M, Dennis ES, Berger F, Peacock WJ, Chaudhury A. 2005. MINISEED3 (MINI3), a WRKY family gene, and HAIKU2 (IKU2), a leucine-rich repeat (LRR) KINASE gene, are regulators of seed size in Arabidopsis. Proceedings of the National Academy of Sciences 102: $17531-17536$.

Maere S, Heymans K, Kuiper M. 2005. BiNGO: A Cytoscape plugin to assess overrepresentation of Gene Ontology categories in Biological Networks. Bioinformatics 21: $3448-3449$.

Marques A, Buijs G, Ligterink W, Hilhorst H. 2018. Evolutionary ecophysiology of seed desiccation sensitivity. Functional Plant Biology 45: 1083.

Matias-Hernandez L, Battaglia R, Galbiati F, Rubes M, Eichenberger C, Grossniklaus U, Kater MM, Colombo L. 2010. VERDANDI is a direct target of the MADS domain ovule identity complex and affects embryo sac differentiation in arabidopsis. The Plant Cell 22: 1702-1715.

Mendes MA, Guerra RF, Castelnovo B, Silva-Velazquez Y, Morandini P, Manrique S, Baumann N, Groß-Hardt R, Dickinson H, Colombo L. 2016. Live and let die: a REM complex promotes fertilization through synergid cell death in Arabidopsis. Development 143: 2780-2790.

Mugal CF, Wolf JBW, Kaj I. 2014. Why time matters: Codon evolution and the temporal dynamics of dN/dS. Molecular Biology and Evolution 31: 212-231.

Pandey S, Nelson DC, Assmann SM. 2009. Two novel GPCR-Type G proteins are abscisic acid receptors in arabidopsis. Cell 136: 136-148.

Park S-Y, Peterson FC, Mosquna A, Yao J, Volkman BF, Cutler SR. 2015. Agrochemical control of plant water use using engineered abscisic acid receptors. Nature 520: 545-548.

Righetti K, Vu JL, Pelletier S, Vu BL, Glaab E, Lalanne D, Pasha A, Patel R V., Provart 
NJ, Verdier J, et al. 2015. Inference of longevity-related genes from a robust coexpression network of seed maturation identifies regulators linking seed storability to biotic defense-related pathways. The Plant Cell 27: tpc.15.00632.

Rosnoblet C, Aubry C, Leprince O, Vu BL, Rogniaux H, Buitink J. 2007. The regulatory gamma subunit SNF4b of the sucrose non-fermenting-related kinase complex is involved in longevity and stachyose accumulation during maturation of Medicago truncatula seeds. Plant Journal 51: 47-59.

Rosvall M, Bergstrom CT. 2008. Maps of random walks on complex networks reveal community structure. Proceedings of the National Academy of Sciences 105: 1118-1123.

Schranz ME, Mohammadin S, Edger PP. 2012. Ancient whole genome duplications, novelty and diversification: the WGD Radiation Lag-Time Model. Current Opinion in Plant Biology 15: 147-153.

Sershen, Berjak P, Pammenter NW, Wesley-Smith J. 2012. The effects of various parameters during processing for cryopreservation on the ultrastructure and viability of recalcitrant zygotic embryos of Amaryllis belladonna. Protoplasma 249: 155-169.

Simão FA, Waterhouse RM, Ioannidis P, Kriventseva E V., Zdobnov EM. 2015. BUSCO: assessing genome assembly and annotation completeness with single-copy orthologs. Bioinformatics 31: 3210-3212.

Song L, Florea L, Langmead B. 2014. Lighter: fast and memory-efficient sequencing error correction without counting. Genome Biology 15: 509.

Steadman KJ, Pritchard HW, Dey PM. 1996. Tissue-specific soluble sugars in seeds as indicators of storage category. Annals of Botany 77: 667-674.

Steber CM. 2001. A role for brassinosteroids in germination in arabidopsis. Plant Physiology 125: $763-769$.

Sun Y, Fan XY, Cao DM, Tang W, He K, Zhu JY, He JX, Bai MY, Zhu S, Oh E, et al. 2010. Integration of brassinosteroid signal transduction with the transcription network for plant growth regulation in arabidopsis. Developmental Cell 19: 765-777.

Terrasson E, Buitink J, Righetti K, Ly Vu B, Pelletier S, Zinsmeister J, Lalanne D, Leprince O. 2013. An emerging picture of the seed desiccome: confirmed regulators and newcomers identified using transcriptome comparison. Frontiers in Plant Science 4: 1-16.

Tunnacliffe A, Wise MJ. 2007. The continuing conundrum of the LEA proteins. Naturwissenschaften 94: 791-812.

Vaistij FE, Gan Y, Penfield S, Gilday AD, Dave A, He Z, Josse E-M, Choi G, Halliday KJ, Graham IA. 2013. Differential control of seed primary dormancy in Arabidopsis 
ecotypes by the transcription factor SPATULA. Proceedings of the National Academy of Sciences 110: 10866-10871.

VanBuren R, Wai CM, Zhang Q, Song X, Edger PP, Bryant D, Michael TP, Mockler TC, Bartels D. 2017. Seed desiccation mechanisms co-opted for vegetative desiccation in the resurrection grass Oropetium thomaeum. Plant Cell and Environment 40: 2292-2306.

Verdier J, Lalanne D, Pelletier S, Torres-Jerez I, Righetti K, Bandyopadhyay K, Leprince O, Chatelain E, Vu BL, Gouzy J, et al. 2013. A regulatory network-based approach dissects late maturation processes related to the acquisition of desiccation tolerance and longevity of Medicago truncatula seeds. Plant Physiology 163: 757-774.

Walker BJ, Abeel T, Shea T, Priest M, Abouelliel A, Sakthikumar S, Cuomo CA, Zeng Q, Wortman J, Young SK, et al. 2014. Pilon: An integrated tool for comprehensive microbial variant detection and genome assembly improvement. PLOS ONE 9: e112963.

Wang Y, Tang H, DeBarry JD, Tan X, Li J, Wang X, Lee T -h., Jin H, Marler B, Guo H, et al. 2012. MCScanX: a toolkit for detection and evolutionary analysis of gene synteny and collinearity. Nucleic Acids Research 40: e49-e49.

Wang M, Verdier J, Benedito VA, Tang Y, Murray JD, Ge Y, Becker JD, Carvalho H, Rogers C, Udvardi M, et al. 2013. LegumeGRN: A gene regulatory network prediction server for functional and comparative studies. PLOS ONE $\mathbf{8}$.

Xu D, Li J, Gangappa SN, Hettiarachchi C, Lin F, Andersson MX, Jiang Y, Deng XW, Holm M. 2014. Convergence of light and ABA signaling on the ABI5 promoter (L-J Qu, Ed.). PLoS Genetics 10: e1004197.

Yang Z. 2007. PAML 4: Phylogenetic analysis by maximum likelihood. Molecular Biology and Evolution 24: 1586-1591.

Ye C, Hill CM, Wu S, Ruan J, Ma Z. 2016. DBG2OLC: Efficient assembly of large genomes using long erroneous reads of the third generation sequencing technologies. Scientific Reports 6: 31900.

Ye C, Ma ZS, Cannon CH, Pop M, Yu DW. 2012. Exploiting sparseness in de novo genome assembly. BMC Bioinformatics 13: $\mathrm{S} 1$.

Yin Y, Cheong H, Friedrichsen D, Zhao Y, Hu J, Mora-Garcia S, Chory J. 2002. A crucial role for the putative Arabidopsis topoisomerase VI in plant growth and development. Proceedings of the National Academy of Sciences 99: 10191-10196.

Zhao T, Holmer R, Bruijn S de, Angenent GC, van den Burg HA, Schranz ME. 2017. Phylogenomic synteny network analysis of MADS-box transcription factor genes reveals lineage-specific transpositions, ancient tandem duplications, and deep positional 
conservation. The Plant Cell: tpc.00312.2017.

\section{Zinsmeister J, Lalanne D, Terrasson E, Chatelain E, Vandecasteele C, Vu BL, Dubois-} Laurent C, Geoffriau E, Signor C Le, Dalmais M, et al. 2016. ABI5 is a regulator of seed maturation and longevity in legumes. The Plant Cell 28: 2735-2754.

\section{Supporting Information}

Figure S1. Hierarchical clustering of expression values.

Figure S2. Phylogenetic tree showing duplication rates of selected species.

Table S1: Gene ontology (GO) enrichment analysis of biological processes in relation to the acquisition of tolerance to water loss and to seed maturation in Medicago truncatula and Castanospermum australe.

Table S2. Genes changing transcript abundance in Castanospermum australe and Medicago truncatula in comparable seed developmental stages.

Table S3. Genes changing transcript abundance in Castanospermum australe and Medicago truncatula in comparable seed developmental stages during final maturation.

Table S4. Protein-coding genes lost in all DS and retained in at least half of the DT species.

Table S5. Castanospermum australe protein-coding genes with $\mathrm{dN} / \mathrm{dS}$ (number of nonsynonymous substitutions per non-synonymous site $(\mathrm{dN})$ in a given period of time divided by the number of synonymous substitutions per synonymous site (dS) in the same period) ratio $\geq 2$.

Figure legends

Figure 1. Castanospermum australe seed and pod developmental stages. MAF: months after flowering. 
Figure 2. Phylome. Reconstruction of the phylome was based on a concatenated alignment of 183 single copy proteins that are present in at least 19 out of the 20 species surveyed. Species names are coloured according to their seed storage category.

Figure 3. Synonymous mutations of duplicated genes and syntenic blocks. Histograms of synonymous mutations (Ks) of duplicated genes (A-F) and average Ks of syntenic blocks (GL). (A-B) Castanospermum australe, (C-D) Lotus japonicus, (E-F) Medicago truncatula, (GH) Glycine max, (I-J) Phaseolus vulgaris, (K-L) Trifolium pratense.

\section{Figure 4. Hypothetical model for brassinosteroid-regulated seed development (adapted} from Jiang et al. (2013)). Red shapes indicate genes that lost synteny in Castanospermum australe compared to Medicago truncatula. Green shapes indicate genes with higher dN/dS in C. australe than in M. truncatula. Blue shapes indicate genes that lost an ortholog, but kept a paralog in C. australe. BR: brassinosteroid. ATBSI1: ACTIVATION-TAGGED BRI1 (BRASSINOSTEROID-INSENSITIVE1)-SUPPRESSOR1. AP2: APETALA 2. ARF2: AUXIN RESPONSE FACTOR 2. BAK1: BRI1-ASSOCIATED RECEPTOR KINASE 1. BRI1: BRASSINOSTEROID INSENSITIVE 1. BRL3: BRASSINOSTEROID INSENSITIVE-LIKE 3. BZR1: BRASSINAZOLE RESISTANT 1. DET2: DE-ETIOLATED 2. IKU: HAIKU. MINI3: MINISEED 3. ROT3: ROTUNDIFOLIA 3. SHB1: SHOEBOX 1.

\section{Table legends}

Table 1. Phenotypic parameters associated with late seed development of Castanospermum australe. Point of attachment refers to tissue attaching the embryo to the seed pod.

Table 2. Overview of assembly, annotation, polymorphism and repeat elements on the Castanospermum australe genome. N50: scaffold size above which 50\% of the total length of the sequence assembly can be found. L90: number of contigs whose summed length contains at least $90 \%$ of the sum of the total length of the sequence assembly. rRNA: ribosomal RNA. snRNA: small nuclear RNA. tRNA: transfer RNA. SNP: single nucleotide polymorphism. INDEL: insertion or deletion of bases in the DNA. SINE: short interspersed nuclear element. LINE: long interspersed nuclear element. LTR: long terminal repeat. L1: LINE-1. RTE: retrotransposable element. hAT: hobo/Ac/Tam3. PIF: P Instability Factor. CMC: CACTA/Mirage/Chapaev. 
bioRxiv preprint doi: https://doi.org/10.1101/665661. The copyright holder for this preprint (which was not peer-reviewed) is the author/funder. All rights reserved. No reuse allowed without permission. 


\section{Figures}

818

819

820

821

822

823

824

825

826

827

828

829

830

831

832

833



Desiccation sensitive

Intermediate

Desiccation tolerant after flowering.
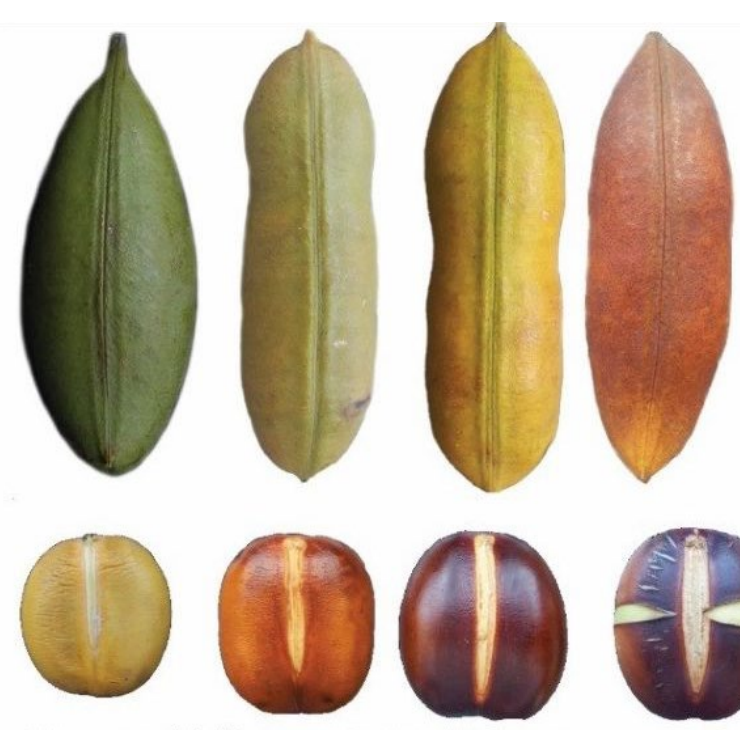

Green Yellow-green Yellow

$$
3 \text { MAF }
$$

$4 \mathrm{MAF}$

$5 \mathrm{MAF}$

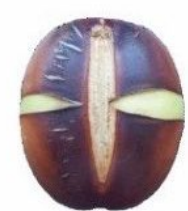

Brown

$6 \mathrm{MAF}$

Figure 1. Castanospermum australe seed and pod developmental stages. MAF: months

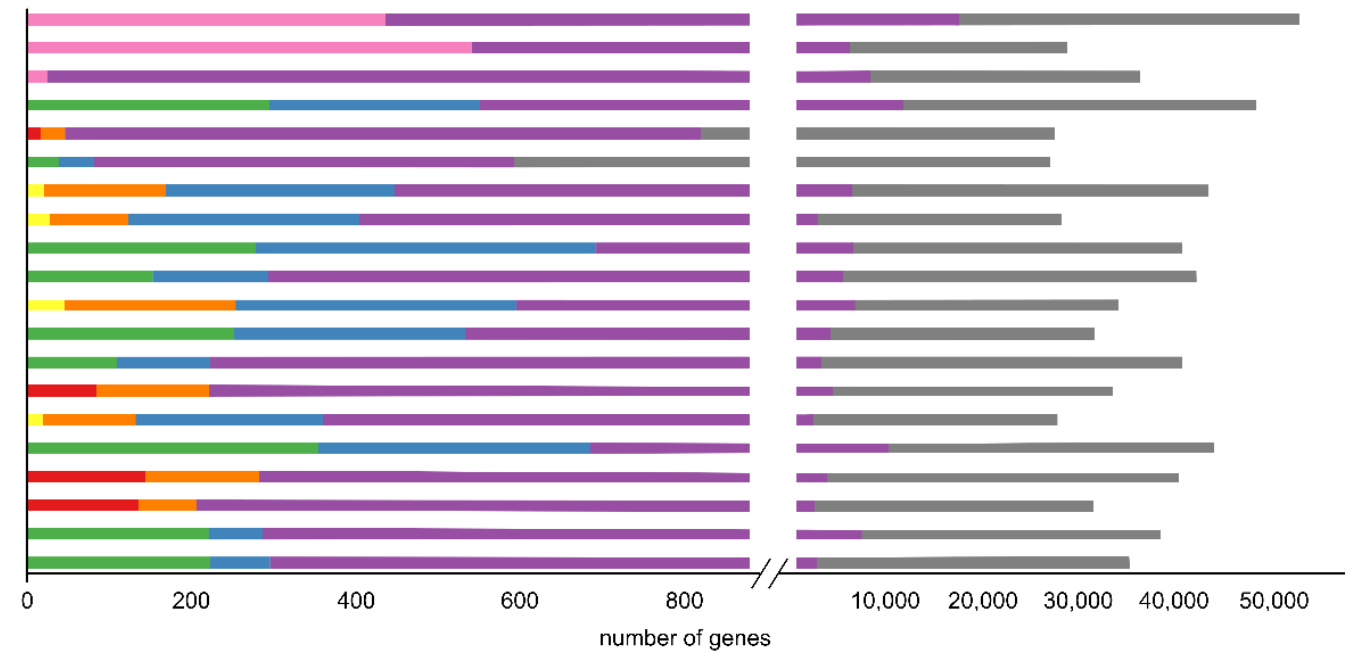

Present only in DS-seeded species

$\square$ Present only in intermediate-seeded species

$\square$ Present only in DT-seeded species $\square$ Present only in DS- and intermediate-seeded species

$\square$ Present only in DT- and intermediate-seeded species $\square$ Present only in outgroups

$\square$ Orphans

$\square$ Total

845 Figure 2. Phylome. Reconstruction of the phylome was based on a concatenated alignment of

846183 single copy proteins that are present in at least 19 out of the 20 species surveyed. Species

847 names are coloured according to their seed storage category. 
Figure 3. Phylogenetic tree showing duplication rates of selected species including species of the subfamilies: Mimosoideae (inside yellow rectangle) and Caesalpinioideae (inside orange rectangle) and Papilionoideae (inside green rectangle). Phylogentic tree based on: Lavin, M., Herendeen, P.S. and Wojciechowski, M.F. (2005) evolutionary rates analysis of leguminosae implicates a rapid diversification of lineages during the tertiary.

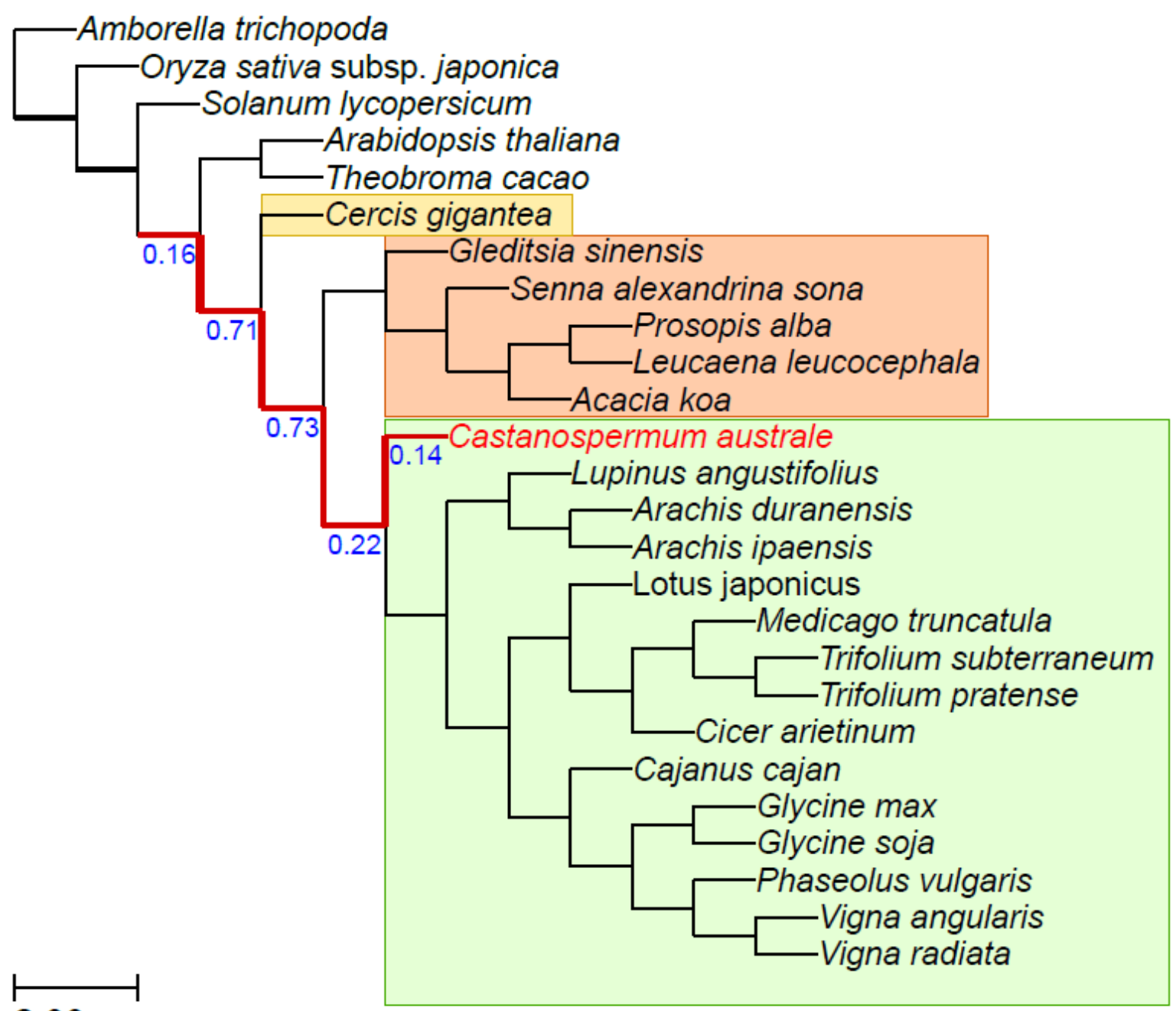



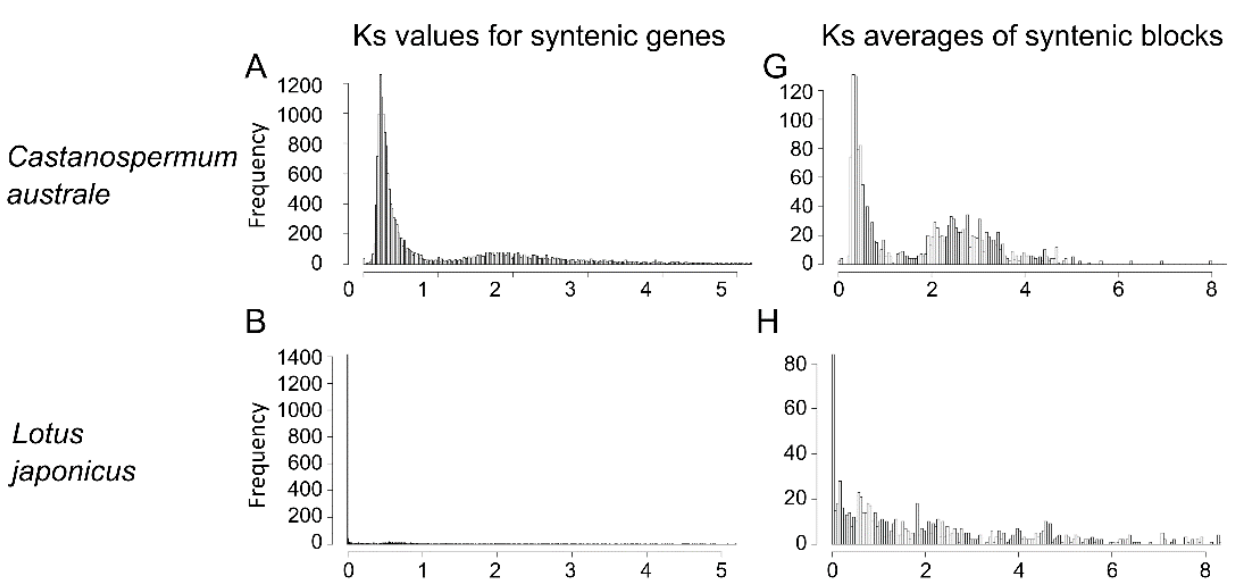

\section{Medicago} truncatula
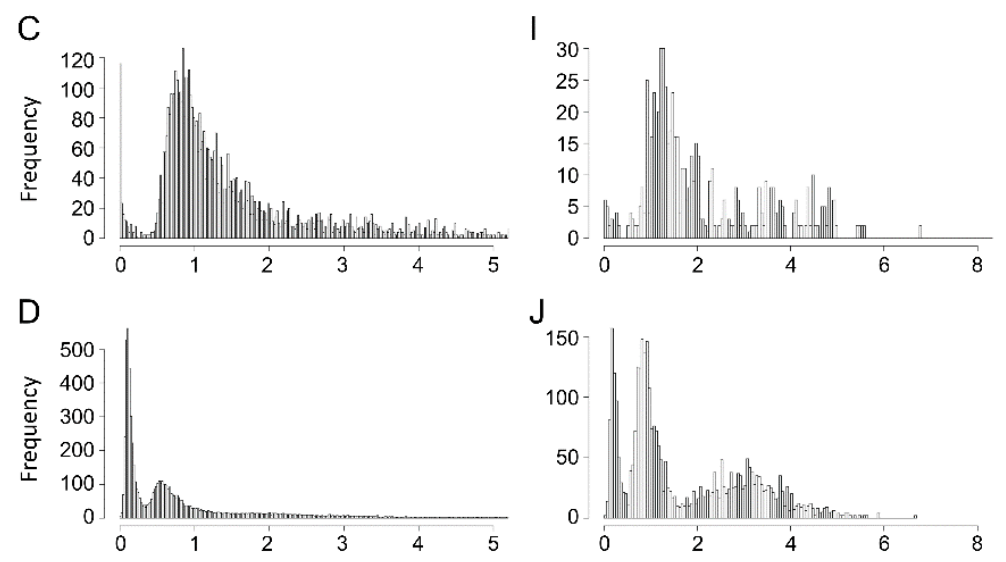

Glycine max
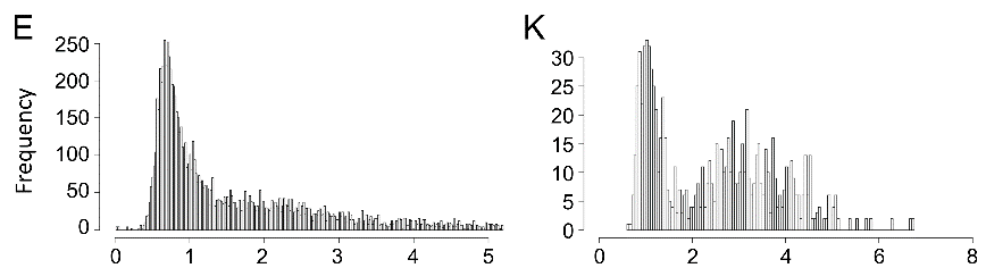

Phaseolus vulgaris
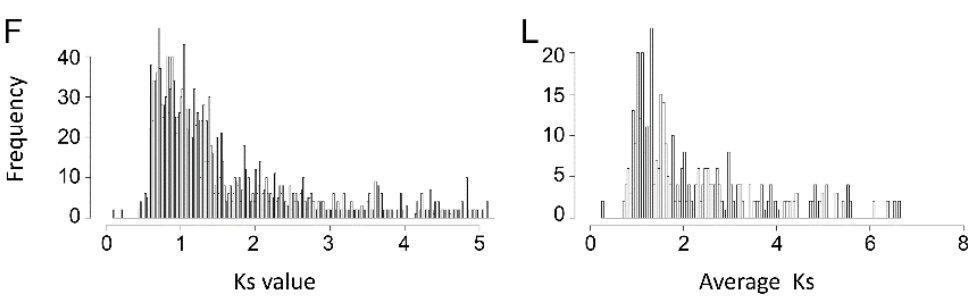

Trifolium pratense

Figure 4. Synonymous mutations of duplicated genes and syntenic blocks. Histograms of synonymous mutations (Ks) of duplicated genes (A-F) and average Ks of syntenic blocks (GL). (A-B) Castanospermum australe, (C-D) Lotus japonicus, (E-F) Medicago truncatula, (GH) Glycine max, (I-J) Phaseolus vulgaris, (K-L) Trifolium pratense. 


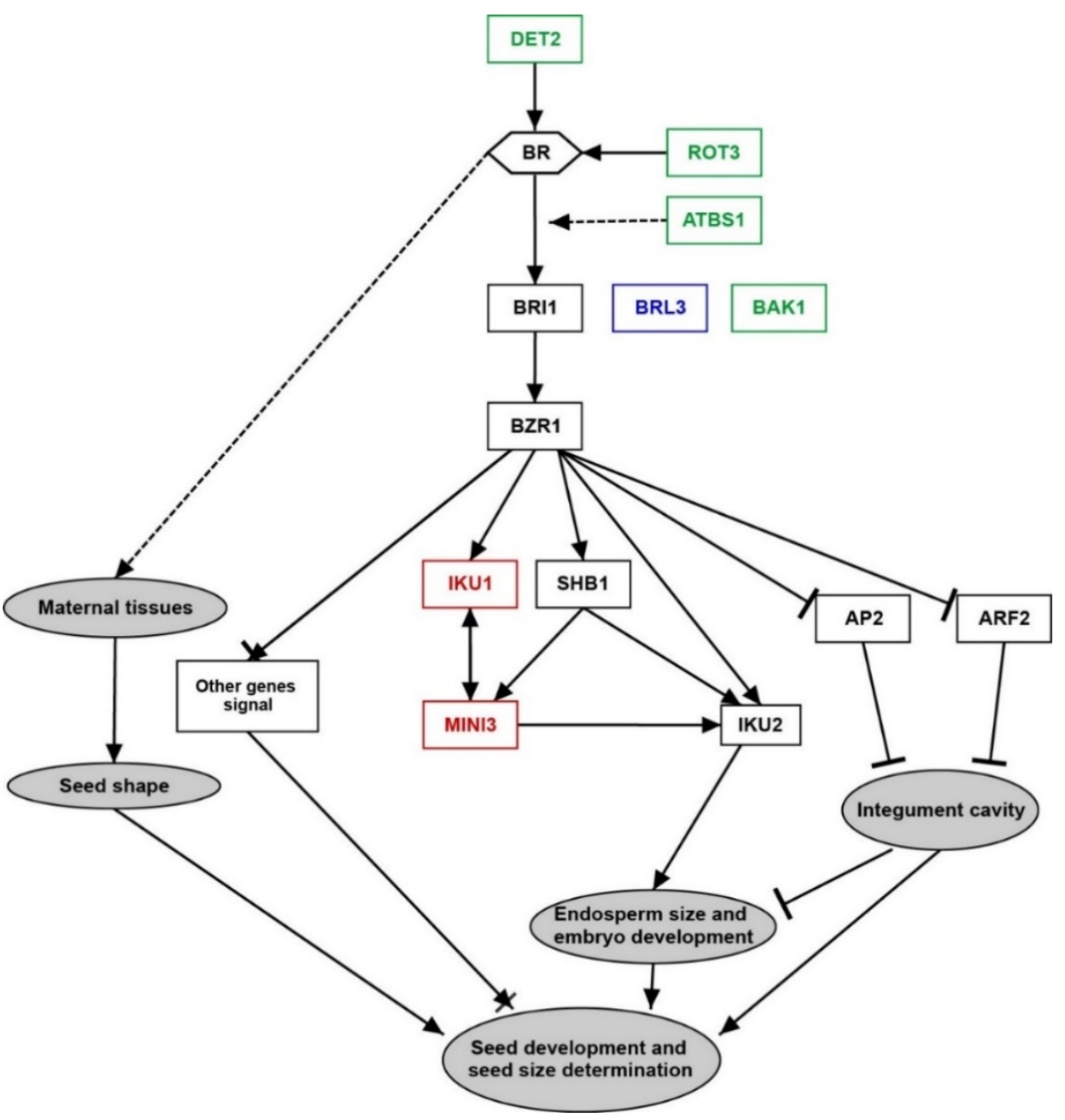

Figure 5. Hypothetical model for brassinosteroid-regulated seed development (adapted

from Jiang et al. (2013)). Red shapes indicate genes that lost synteny in Castanospermum australe compared to Medicago truncatula. Green shapes indicate genes with higher dN/dS in C. australe than in M. truncatula. Blue shapes indicate genes that lost an ortholog, but kept a paralog in C. australe. BR: brassinosteroid. ATBSI1: ACTIVATION-TAGGED BRI1 (BRASSINOSTEROID-INSENSITIVE1)-SUPPRESSOR1. AP2: APETALA 2. ARF2: AUXIN RESPONSE FACTOR 2. BAK1: BRI1-ASSOCIATED RECEPTOR KINASE 1. BRI1: BRASSINOSTEROID INSENSITIVE 1. BRL3: BRASSINOSTEROID INSENSITIVE-LIKE 3. BZR1: BRASSINAZOLE RESISTANT 1. DET2: DE-ETIOLATED 2. IKU: HAIKU. MINI3: 
Table 1. Phenotypic parameters associated with late seed development of Castanospermum australe. Point of attachment refers to tissue attaching the embryo to the seed pod.

\begin{tabular}{|c|c|c|c|c|c|c|c|c|c|}
\hline \multirow[t]{2}{*}{ Pod stage } & & \multicolumn{2}{|c|}{ Green } & \multicolumn{2}{|c|}{ Yellow-green } & \multicolumn{2}{|c|}{ Yellow } & \multicolumn{2}{|c|}{ Brown } \\
\hline & & average & SE & average & $\mathrm{SE}$ & average & $\mathrm{SE}$ & average & SE \\
\hline Whole seed mass (g) & & 19.236 & 0.586 & 29.618 & 0.959 & 38.037 & 0.867 & 40.389 & 0.720 \\
\hline Seed coat & $\operatorname{mass}(\mathrm{g})$ & 2.496 & 0.082 & 1.342 & 0.039 & 1.210 & 0.032 & 1.228 & 0.037 \\
\hline \multicolumn{2}{|c|}{ Germination $(\%)$ of axes on MS media } & 93 & & 100 & & 100 & & 100 & \\
\hline \multicolumn{2}{|l|}{ Time (days) to reach $50 \%$ germination } & 25 & & 8 & & 8 & & 8 & \\
\hline \multirow[t]{2}{*}{ Water content ( $\mathrm{gH}_{2} \mathrm{O} \mathrm{g}^{-1}$ dry mass) } & axes & 4.030 & 0.149 & 3.458 & 0.113 & 2.412 & 0.061 & 2.426 & 0.045 \\
\hline & cotyledons & 5.084 & 0.209 & 3.255 & 0.231 & 1.559 & 0.073 & 1.783 & 0.122 \\
\hline \multirow{5}{*}{$\begin{array}{l}\text { Water content ( } \mathrm{gH}_{2} \mathrm{O} \mathrm{g}^{-1} \text { dry mass) at } \\
\text { which } 50 \% \text { loss of viability occurs } \\
\text { Glucose ( } \mathrm{mg} \mathrm{g}^{-1} \text { dry mass) }\end{array}$} & axes & 0.448 & 0.021 & 0.342 & 0.016 & 0.228 & 0.012 & 0.221 & 0.009 \\
\hline & cotyledons & 0.820 & 0.026 & 0.735 & 0.021 & 0.766 & 0.021 & 0.700 & 0.027 \\
\hline & axes & 0.391 & 0.144 & 1.545 & 0.194 & 1.162 & 0.366 & 1.088 & 0.410 \\
\hline & cotyledons & 0.829 & 0.719 & 0.528 & 0.228 & 0.261 & 0.106 & 0.661 & 0.064 \\
\hline & point of attachment & & & 0.515 & 1.486 & 0.245 & 0.193 & 0.450 & 0.257 \\
\hline \multirow{2}{*}{ Fructose (mg g $\mathrm{g}^{-1}$ dry mass) } & axes & 0.243 & 0.218 & 0.648 & 0.063 & 0.912 & 0.456 & 0.100 & 0.173 \\
\hline & cotyledons & 0.491 & 0.438 & 0.343 & 0.069 & 0.336 & 0.033 & 0.359 & 0.115 \\
\hline \multirow[t]{3}{*}{ Sucrose (mg g ${ }^{-1}$ dry mass) } & axes & 40.615 & 6.792 & 81.402 & 3.842 & 89.790 & 13.852 & 82.760 & 6.089 \\
\hline & cotyledons & 41.428 & 18.578 & 25.854 & 6.820 & 63.297 & 3.108 & 58.342 & 12.720 \\
\hline & point of attachment & & & 1.858 & 0.515 & 0.783 & 0.319 & 1.434 & 1.312 \\
\hline \multirow[t]{3}{*}{ Stachyose (mg g ${ }^{-1}$ dry mass) } & axes & 1.947 & 0.644 & 1.767 & 0.179 & 1.139 & 0.116 & 1.043 & 0.170 \\
\hline & cotyledons & 2.948 & 1.705 & 0.154 & 0.135 & 0.112 & 0.043 & 0.128 & 0.026 \\
\hline & point of attachment & & & 1.188 & 1.858 & 1.948 & 0.167 & 1.288 & 0.281 \\
\hline \multirow[t]{3}{*}{ Raffinose (mg g ${ }^{-1}$ dry mass) } & axes & 1.990 & 0.217 & 0.546 & 0.184 & 0.000 & 0.000 & 0.205 & 0.354 \\
\hline & cotyledons & 1.576 & 0.309 & 0.328 & 0.567 & 0.639 & 0.619 & 0.531 & 0.477 \\
\hline & point of attachment & & & 0.000 & 0.950 & 0.000 & 0.000 & 0.000 & 0.000 \\
\hline \multirow[t]{4}{*}{ ABA content (pmol g-1 dry mass) } & axes & 8556.94 & 62.50 & 7676.37 & 42.89 & 817.89 & 82.08 & 444.95 & 7.65 \\
\hline & cotyledons & 18393.12 & 335.31 & 16153.81 & 160.86 & 4311.68 & 188.76 & 1860.64 & 35.90 \\
\hline & point of attachment & 9404.77 & 101.66 & 11710.72 & 265.05 & 19400.48 & 181.23 & 24172.84 & 574.80 \\
\hline & seed coat & 9175.61 & 330.12 & 32259.10 & 115.59 & 15779.45 & 125.60 & 23380.20 & 216.08 \\
\hline
\end{tabular}


Table 2. Overview of assembly, annotation, polymorphism and repeat elements on the Castanospermum australe genome. N50: scaffold size above which 50\% of the total length of the sequence assembly can be found. L90: number of contigs whose summed length contains at least $90 \%$ of the sum of the total length of the sequence assembly. rRNA: ribosomal RNA. snRNA: small nuclear RNA. tRNA: transfer RNA. SNP: single nucleotide polymorphism. INDEL: insertion or deletion of bases in the DNA. SINE: short interspersed nuclear element. LINE: long interspersed nuclear element. LTR: long terminal repeat. L1: LINE-1. RTE: retrotransposable element. hAT: hobo/Ac/Tam3. PIF: $\mathrm{P}$ Instability Factor. CMC: CACTA/Mirage/Chapaev.

\begin{tabular}{lccccc}
\hline Assembly & Number & N50 (Kb) & $\begin{array}{c}\text { L90 } \\
(\mathbf{k b})\end{array}$ & $\begin{array}{c}\text { Total } \\
\text { length }\end{array}$ & Alignment rate \\
\hline Contigs & 1,210 & 761.1 & - & - & - \\
Scaffolds & 1,027 & 832.6 & 495 & $381.7 \mathrm{Mb}$ & $97.7 \%$
\end{tabular}

\begin{tabular}{lcccc}
\hline Annotation & Number & $\begin{array}{c}\text { Mean lengh } \\
\text { (bp) }\end{array}$ & Density & $\begin{array}{c}\text { Genome } \\
\text { percentage }\end{array}$ \\
\hline Protein coding genes & 29,124 & 4814.9 & - & $36.7 \%$ \\
Exons & 180,329 & 232.7 & 6.2 exons/gene & $11.1 \%$ \\
Introns & 141,174 & 696.1 & 5.0 introns/gene & $25.6 \%$ \\
rRNA & 149 & 623.83 & - & $0.0 \%$ \\
snRNA & 60 & 121.72 & - & $0.0 \%$ \\
tRNA & 310 & 75.55 & - & $0.0 \%$ \\
Transposable & 110,949 & - & - & $15.5 \%$ \\
elements & & & &
\end{tabular}

\begin{tabular}{lcc}
\hline Polymorphisms & Number & Density \\
\hline SNPs & 352,963 & $0.92 \mathrm{~kb}^{-1}$ \\
INDELS & 328,918 & $0.86 \mathrm{~kb}^{-1}$ \\
Multi-allelic sites & 6,328 & $0.02 \mathrm{~kb}^{-1}$ \\
& & \\
\hline Repeat genus & Length & Abundant species \\
\hline SINE & $118,770(0.05 \%)$ & L1, RTE \\
LINE & $3,834,244(1.6 \%)$ & $h A T$, PIF, CMC \\
DNA transposon & $11,400,282(4.8 \%)$ & Gypsy, Copia \\
LTR & $42,305,056(17.84)$ & - \\
Unclassified/Simple & $177,648,468(74.9 \%)$ &
\end{tabular}


